\title{
The effect of increased fruit and vegetable consumption on systolic and diastolic blood pressure in six randomized controlled fruit and vegetable intervention trials: a pooled analysis
}

\author{
N. Elsahoryi ${ }^{1}$, C.C. Patterson ${ }^{1,2}$, M.C. McKinley ${ }^{1,2}$, C.E. Neville ${ }^{1}$, F.R. Baldrick ${ }^{1}$, C. Mulligan ${ }^{3}$, \\ D.O. McCall ${ }^{4}$, D.R. McCance ${ }^{5}$, J.D. Edgar ${ }^{6}$, J.S. Elborn ${ }^{7}$, I. Wallace ${ }^{5}$, C. McEvoy ${ }^{1}$, S. Hunter ${ }^{5}$, \\ R.L. Noad ${ }^{4}$, C. Rooney ${ }^{1}$, P.P. McKeown ${ }^{8}$, I.S. Young ${ }^{1,2}$ and J.V. Woodside ${ }^{1,2}$ \\ ${ }^{1}$ Centre for Public Health, School of Medicine, Dentistry and Biomedical Sciences, Institute of Clinical Science B, \\ Queen's University Belfast, Belfast, BT12 6BJ, UK, \\ ${ }^{2}$ UKCRC Centre of Excellence for Public Health (Northern Ireland), Queen's University Belfast, Belfast, BT12 6BJ, UK, \\ ${ }^{3}$ Diabetes Department, Ulster Hospital, South Eastern Trust, Belfast, BT16 1RH, \\ ${ }^{4}$ Belfast Health and Social Care Trust (BHSCT), Belfast, BT9 7AB, \\ ${ }^{5}$ Regional Centre for Endocrinology, BHSCT, Belfast, BT12 6BA, \\ ${ }^{6}$ Regional Immunology Service, BHSCT, Belfast, BT12 6BA, \\ ${ }^{7}$ Centre for Experimental Medicine, Queen's University Belfast, Belfast, BT9 $7 \mathrm{JL}$ and \\ ${ }^{8}$ Centre for Medical Education, Queen's University Belfast, Belfast, BT9 $7 B L$
}

Observational evidence consistently shows that increasing fruit and vegetable (FV) consumption is associated with reduced risk of chronic diseases, including hypertension, as these foods are important sources of micronutrients, dietary fibre, and phytochemicals and are low in energy density. Assessment of dietary intake is difficult and there are many sources of error, while observational studies cannot demonstrate cause and effect. The aim of this analysis was to use data from six FV trials to examine the effect of increasing FV intake on systolic blood pressure (SBP) and diastolic blood pressure (DBP).

The six randomized controlled trials $\left(\mathrm{FVD}^{(1)}, \mathrm{FAVRIT}^{(2)}, \mathrm{DISCO}^{(3)}, \mathrm{PPHIT}^{(4)}, \mathrm{FIRST}^{(5)}\right.$ and $\mathrm{ADIT}^{(6)}$ ) were carried out in a range of populations but were of similar duration and used comparable methods to achieve dietary change and for outcome assessment. FV were supplied free of charge to participants and self-reported intake was assessed via either diet history or food diary. SBP and DBP were recorded using automated sphygmomanometers.

Changes in SBP and DBP were each regressed on change in self-reported FV intake, the slope providing an estimate of the change in each variable associated with a one portion change in self-reported FV intake. The slopes and their standard errors from the six trials were then inputted into Revman v. 5.3 and a random effects model used to pool results. Tests for heterogeneity of slopes between studies were performed and forest plots generated, as shown below. A p-value of $<0.05$ was considered statistically significant for testing the pooled results.

The pooled regression results for the six trials showed that there was no significant decrease in either SBP or DBP per one portion increase in FV, although there was evidence of significant heterogeneity across studies $\left(I^{2}=73 \%, 43 \%\right.$ respectively).

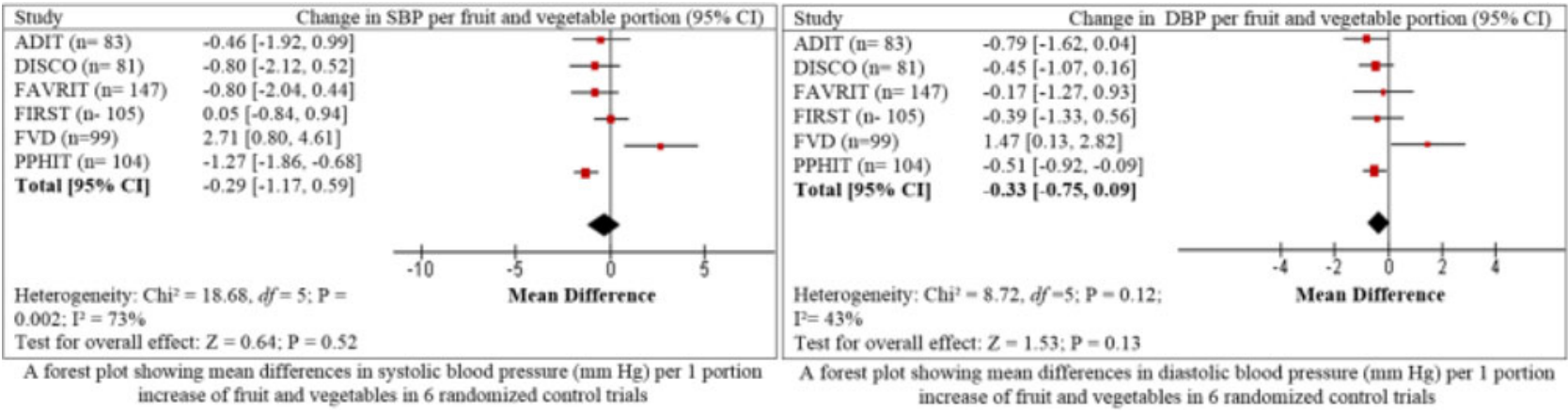

In conclusion, pooled regression analysis of six FV trials showed no statistically significant effect of increased FV intake on SBP or DBP, with significant between study heterogeneity.

1. Daniels J, Mulligan C, McCance D, Woodside J et al. (2014) Am J Clin Nutr 96, 1429-36.

2. McCall DO, McGartland CP, McKinley MC et al. (2009) Circulation 119, 2153-60.

3. Baldrick FR, Elborn JS, Woodside J V et al. (2012) Eur Respir J 39, 1377-84.

4. Noad RL, Rooney C, McCall D et al. (2016) Heart J 102, 1371-9.

5. Wallace I, McEvoy C, Hunter S et al. (2013) Diabetes Care J 36, 3888-3896.

6. Gibson A, Edgar JD, Neville CE et al. (2012) Am J Clin Nutr 96, 2409-2422. 